\title{
A crosslinguistic study of the acquisition of time words in English- and German- speaking children
}

\author{
Katherine Williams (kwilliams03@utexas.edu) \\ Department of Psychology, 108 E. Dean Keeton Stop A8000 \\ Austin, TX \\ Gabriela Markova (gabriela.markova@univie.ac.at) \\ Faculty of Psychology, Liebiggasse 5, 1010 \\ Vienna, Austria
}

\author{
Anna Bánki (anna.banki@univie.ac.at) \\ Faculty of Psychology, Liebiggasse 5, 1010 \\ Vienna, Austria \\ Stefanie Hoehl (stefanie.hoehl@univie.ac.at) \\ Faculty of Psychology, Liebiggasse 5, 1010 \\ Vienna, Austria
}

\section{Katharine Tillman (ktillman@utexas.edu) \\ Department of Psychology, 108 E. Dean Keeton Stop A8000}

\section{Austin, TX}

\begin{abstract}
Unlike English, German contains single words for "the day after tomorrow" (übermorgen) and "the day before yesterday" (vorgestern). How might these cross-linguistic differences influence children's acquisition of time words? Prior work shows that English-speaking preschoolers learn the deictic status of time words (e.g., yesterday was in the past) long before learning their precise temporal locations (e.g., yesterday was exactly one day ago). Here we ask whether the set of time words influences children's understanding of proximal (yesterday/tomorrow) and distal (day before yesterday/day after tomorrow) terms. English- and Germanspeaking 3- to 7-year-olds $(N=253)$ marked the temporal location of each term relative to today on a calendar template. While children in both language groups demonstrated equal knowledge of deictic status, German speakers were more likely to have precise meanings for proximal and distal items, suggesting that having more alternative time words available may help narrow the scope of children's meanings.
\end{abstract}

Keywords: cognitive development; temporal cognition; abstract concepts; cross-linguistic comparison; word learning

\section{Introduction}

As adults, we have a highly structured concept of time that underpins our interpretation of past memories, day-to-day schedules, and future planning. How young children experience time, think about time, and learn to encode time in an adult-like way is a current topic of investigation among cognitive development and language acquisition researchers. Although children first begin to produce time words around age 2 or 3, describing and measuring time in an adult-like way using temporal language, conventional units of time, and spatial artifacts, develops substantially later (Ames, 1946; Friedman \& Kemp, 1998; Grant \& Suddendorf, 2011). This raises questions about why there is a lag between production and comprehension, how and when children converge on precise meanings, and whether particular linguistic cues aid in their acquisition of time words. In the current study, we conduct a cross-linguistic comparison to examine how different features of language impact early time-word learning.

Deictic time words, like yesterday and tomorrow, encode the temporal relationship between a given event and the present. Thus, their reference depends on the context in which they are said, posing a particular challenge for language learners. Children produce words like tomorrow as early as age 3 (and typically produce tomorrow earlier than yesterday, see Ames, 1946; Frank et al., 2016; Grant \& Suddendorf, 2011) but do not demonstrate adult-like comprehension until at least age 7 (Hudson \& Mayhew, 2011; Tillman et al., 2017). Acquiring adult-like meanings for deictic time words may also be challenging for young children because their meanings are multifaceted, including information about whether the event is in the past or future (i.e., deictic status) and the distance of the event from the present (i.e., remoteness).

Although it is widely known that children initially struggle to comprehend deictic time words, there has been variability across methods used to assess children's knowledge of their meanings and in researchers' conclusions about the age at which children acquire them. Some studies have focused specifically on children's discrimination of yesterday and tomorrow. For example, when asked to select a toy "from yesterday" or "for tomorrow," 3-year-olds made fewer errors in response to yesterday, while 4-year-olds made highly accurate selections in response to both yesterday and tomorrow, demonstrating their knowledge that yesterday refers to the past and tomorrow refers to the future (Harner, 1975). Nonetheless, after hearing a puppet describe an action that happened today, 5-year-olds in a recent study were still more accurate in answering questions about what happened yesterday relative to questions about what will happen tomorrow (Zhang and Hudson, 2018). While neither of these studies asked whether children know precisely how far into the past or future the terms apply, other work indicates that children's early understanding of time words is impacted by their remoteness from the present. For example, using a timeline task, Hudson and Mayhew (2011) found that 5- to 6year-olds were less accurate when indicating far distances (e.g., two weeks ago) than near distances (e.g., tomorrow). Seven-year-olds, on the other hand, were highly accurate across temporal distances. 
Other findings suggest that children systematically learn some aspects of time-word meaning before others. Tillman and colleagues (2017) asked 3- to 7-year-old Englishspeakers to indicate where several time words belonged on a timeline extending from the past (infancy) to the future (adulthood). Most children were able to accurately place items to the left (past) vs. right (future) of "now" by age 5 . This knowledge of deictic status emerged in tandem with knowledge of the relative ordering of the terms, i.e. that last week is farther in the past than yesterday. However, knowledge of temporal remoteness emerged almost two years later. Here, we use the term 'precise meaning' to refer to knowledge of both deictic status and remoteness.

Interestingly, the components of time-word meanings that first emerge in English-speakers could be linked to linguistic cues available to them in their speech input. For example, tense markings could be used to infer deictic status (e.g., "I danced yesterday" indicates that yesterday is a time in the past). Information about remoteness, on the other hand, cannot be gleaned from tense cues in English. ${ }^{1}$ If children are using the linguistic context to infer meaning, this might explain why deictic status emerges prior to knowledge of remoteness. Similarly, knowledge of the relative ordering of terms could be inferred from contrastive uses and order-ofmention in discourse (e.g., "Last week she took the train, but yesterday she walked home"), but, again, remoteness cannot. Although previous results from English-speakers suggest that linguistic cues play an important role in bootstrapping timeword acquisition, this hypothesis has not yet been directly tested.

One way of exploring what linguistic cues might contribute to time-word learning is to compare learning in speakers of different languages. Although much of the research on children's acquisition of deictic time words has been conducted in English, languages vary in the sets of time words they contain and how their different facets of meaning (e.g., deictic status and remoteness) are encoded. This raises the question of whether there are cross-linguistic differences in time-word acquisition.

Here we compare the acquisition of deictic time words in English and German. Like in English, grammatical tense in German provides a linguistic cue to the deictic status of time words. Both English and German have a single term to refer to a time exactly one day in the future (tomorrow/morgen) or exactly one day in the past (yesterday/gestern). German has additional compound words that refer to a time two days in the future (übermorgen) or two days in the past (vorgestern), but English speakers must use an entire phrase to indicate those times (e.g., "the day before yesterday"). ${ }^{2}$ Thus, we consider German to have a larger set of deictic time words. Here we ask whether these differences between English and

\footnotetext{
${ }^{1}$ This is not the case in all languages. For example, Inuktitut, an Inuit language spoken in Alaska and northern Canada, contains different tenses that are used to discuss events in the near vs. far past and future (Swift, 2008).

2 Interestingly, while Old English also contained the terms overmorrow and ereyesterday, they are no longer used.
}

German could lead to differences in children's acquisition of time words, and, more specifically, differences in their knowledge of each terms' deictic status and remoteness.

We asked two related questions about German- and English-speakers' knowledge of deictic time words. The first question was whether we would find a difference in children's acquisition of the distal terms that are lexicalized in German but not in English. On the one hand, if it is simpler to learn the meaning of a word (albeit a compound one) that means "exactly two days ago," than a complex phrase involving both a sequential time word (before/after) and a deictic time word (tomorrow/yesterday), we would expect German speakers to have an advantage on these items. On the other hand, learning prefixes like vor- and über-could be equally challenging. Moreover, if the greater variation in how the concept of "exactly two days ago" is expressed across languages reflects the increased difficulty of the concept itself, we would not necessarily expect to see a languagegroup difference in comprehension.

A second question was whether we would find a difference in children's acquisition of the proximal terms lexicalized in both languages. If individual time words are learned independently (i.e., by mapping each one onto a nonverbal event representation), we would not expect to see a languagegroup difference. However, prior work on children's acquisition of duration words suggests that these terms are not learned by mapping them onto perceptual representations of time, but, instead, by inferring their relationship to other time words (i.e., second $<$ minute $<$ hour; see Tillman \& Barner, 2015). On this account, the meaning of each individual term is constrained by children's knowledge of the entire set. Consistent with this, as mentioned above, children appear to learn the relative ordering of deictic time words prior to learning their remoteness (Tillman et al., 2017).

If knowledge of other time words in the set influences the acquisition of deictic time words, there are at least two possible patterns of results we might find. One is that German-speaking children have a disadvantage because they have a larger set of terms to learn. Another possibility is that having access to more tightly spaced distal terms might help constrain children's hypotheses about the scope of more proximal terms. On this hypothesis, if a German-speaker knows a word for "exactly two days ago," they might also figure out that yesterday means "exactly one day ago" sooner than an English-speaker whose closest lexical alternative is approximately six days away (i.e., last week). ${ }^{3}$

Lastly, we consider the possibility that the cross-linguistic differences will have differential effects on children's knowledge of different facets of meaning. In particular, if children are using the syntactic structure of language (e.g., tense) to acquire knowledge of deictic status, as prior studies

\footnotetext{
${ }^{3}$ This difference is further complicated by the fact that the term 'last week' in English does not always refer to a time point one week in the past. Instead, the remoteness of this term may be dependent on the day of the week the utterance is spoken. For example, on a Monday, "last week" could refer to the previous Friday, only 3 days ago.
} 
have proposed, we would not expect to find cross-linguistic differences in children's ability to assign items to the past or future. This is because both languages have similar tense systems, including an inflectional past tense but no inflectionally marked future tense (Dryer et al., 2013). To test these questions, we introduce a novel calendar task in which children place proximal (yesterday/tomorrow) and distal (day before yesterday/day after tomorrow) terms in one of seven contiguous squares representing the days of the week. We asked German- and English-speaking children aged 3-7 to complete the calendar task to test their knowledge of (1) the deictic status of each term and (2) the precise meaning of each term (e.g., that yesterday is exactly one day before today).

\section{Method}

\section{Participants}

A total of 128 English-speakers (4 3-year-olds, 45 4-yearolds, 42 5-year-olds, 29 6-year-olds, 8 7-year-olds) recruited from the United States in Austin, Texas, and 125 Germanspeakers (6 3-year-olds, 44 4-year-olds, 41 5-year-olds, 186 year-olds, 16 7-year-olds) recruited from Vienna, Austria, participated in the study. Four additional English-speaking children were excluded due to experimenter error. Participants were tested in their native language by experimenters who were also native speakers. All study procedures were IRB-approved.

\section{Materials}

Unlike the previously-used timeline task (Tillman et al., 2017), the calendar task is a novel, discontinuous measure in which children's responses are constrained to individual squares with precise distances from today. Thus, children's knowledge of each target word's deictic status and remoteness can be precisely measured and scored. Study materials included a sheet of 8 rows of 7 contiguous squares (see Figure 1) and five colors of round label stickers.
A)

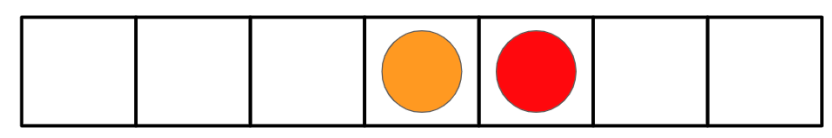

B)

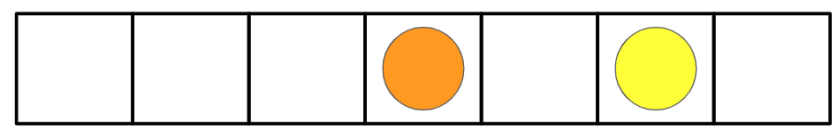

Figure 1: Correct placement for A) tomorrow (red sticker) and B) the day after tomorrow (yellow sticker). On each trial, the orange sticker represents today, and participants select the placement for the target item, e.g. tomorrow.

\section{Procedure}

Children were tested one-on-one. The experimenter began the session by asking the child to recite the days of the week. The experimenter then placed the first row of squares in front of the participant and labeled each square with the days of the week (verbally from left to right). Each trial of the calendar task began with an empty row of squares. The experimenter first placed the orange sticker in the center square to represent today, then gave the child a sticker to place in another square (e.g., "If this one is today, can you put the red sticker in the square for tomorrow"). Different colored stickers were used for each target: yesterday/gestern, tomorrow/morgen, the day after tomorrow/übermorgen, and the day before yesterday/vorgestern. Children who did not place the sticker in the correct square on the first try were presented with a second row and a follow-up forced choice between the proximal and distal squares on the correct side of today, e.g. in the case of yesterday or the day before yesterday: Is it this one [square 3] or this one [square 2]. ${ }^{4}$ Trial order was counterbalanced.

Finally, children were asked five verbal questions about the calendar system: "which day comes after/before today, tomorrow or yesterday?", "what day of the week is it today?", "today is [name of the day], what day of the week was it yesterday/will it be tomorrow?" Children also completed a separate task involving a continuous timeline, either before or after the calendar task. Due to space limitations, we will not discuss results from the timeline task and verbal questions.

\section{Coding and Analyses}

Each row of squares represented time extending three days in the past to three days in the future, from today. On each trial, the experimenter placed a sticker for today in the center (square 4). The correct squares for

\footnotetext{
4 These data are not included in the present report. All data reported in the Results reflect the child's initial sticker placement.
} 

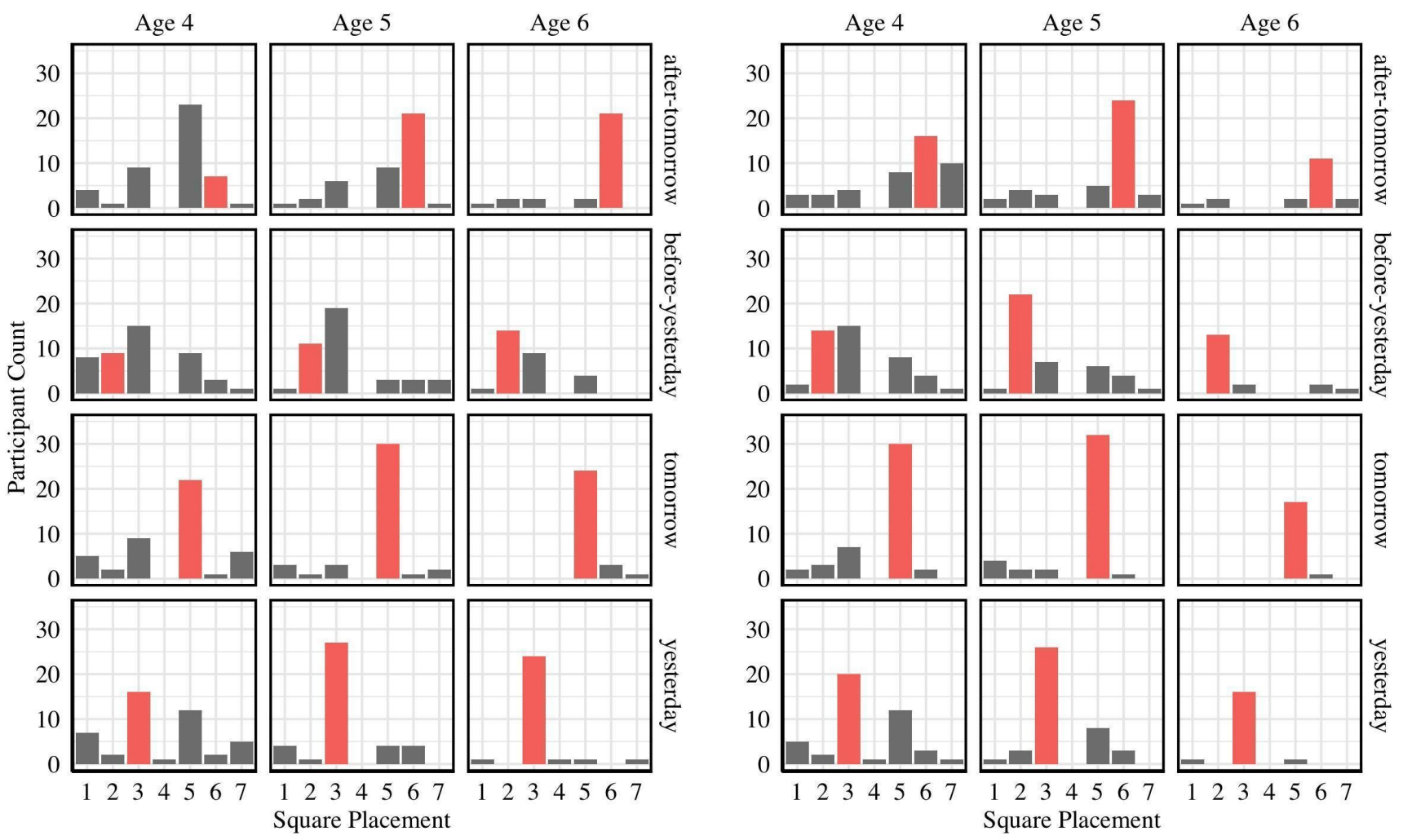

Figure 2: Sticker placements by English- (45 4-year-olds, 42 5-year-olds, 29 6-year-olds) and German-speaking (44 4-yearolds, 41 5-year-olds, 18 6-year-olds) children for each item. Red bars indicate the correct placement.

before-yesterday, ${ }^{5}$ yesterday, tomorrow, and after-tomorrow were $2,3,5$, and 6 , respectively. To assess if children have precise meanings for each term -- including both their deictic status and remoteness -- we coded whether they placed the corresponding sticker in the correct square. To assess children's knowledge of deictic status, we coded whether they correctly placed stickers to the right or left of today. For example, in the assessment of deictic status, a sticker placement for tomorrow was coded as correct (1) if it was placed in squares 5-7 and incorrect (0) if it was placed in squares 1-3.

We conducted mixed-effects logistic regressions using the lme4 package in $R$ (Bates et al., 2015). We treat age as a continuous, scaled variable in all models and include a random intercept for subjects; all fixed effects were effect coded with predictors centered around $0[-1,1]$. We performed Wald chi-square tests from type-III analysis-ofvariance tables using the car package (Fox \& Weisberg, 2019) to determine whether models including each factor of interest provided a significantly better fit to the data than reduced models. We included all data $(N=$

\footnotetext{
5 Throughout the methods and results we abbreviate the day before yesterday and the day after tomorrow to before-yesterday and after-tomorrow, respectively.
}

$253)$ in the models, but we omit 3-year-olds $(n=10)$ and 7year-olds $(n=24)$ from figures due to the small sample sizes in these age groups.

\section{Results}

When asked to recite the days of the week, $65 \%$ of Englishspeakers (including $40 \%$ of 4 -year-olds, $76 \%$ of 5-year-olds, and $79 \%$ of 6-year-olds) named all 7 days in order without help from the experimenter. In contrast, only $28 \%$ of German speakers (including $5 \%$ of 4 -year-olds, $33 \%$ of 5 -year-olds, and $28 \%$ of 6 -year-olds) did so.

The frequency distribution of children's sticker placements for each item is shown in Figure 2. To investigate crosslinguistic differences in children's developing knowledge of time words, we conducted two primary analyses. First, we assessed children's knowledge of the deictic status of both proximal and distal terms. Second, we assessed their knowledge of the precise meanings (i.e., remoteness from the present and deictic status) of these words. 


\section{Knowledge of Deictic Status}

To assess knowledge of deictic status, we asked whether items were correctly placed to the right or left of today. We first considered whether language spoken (German vs. English), item, and/or age predicted children's correct placement of terms in the past or future. The model revealed a main effect of age $\left(\beta=0.91, p<.01 ; \chi^{2}(1)=52.9, p<.01\right)$, but we did not find evidence of a main effect of item $\left(\chi^{2}(3)=\right.$ $1.87, p=.599)$ or language $\left(\chi^{2}(1)=0.63, p=.429\right)$. Older children in both languages were more likely to correctly assign items to the past or future than younger children (see Figure 3). The mean percentage of trials in which children correctly assigned items increased with age: $48 \%$ (95\% CI $[39.5,55.5])$ for 3-year-olds, $68 \%[65.8,70.7]$ for 4-yearolds, $78 \%$ [75.5, 80.1] for 5-year-olds, $90 \%$ [88.3, 92.6] for 6 -year-olds, and $97 \%[95.1,98.7]$ for 7 -year-olds.

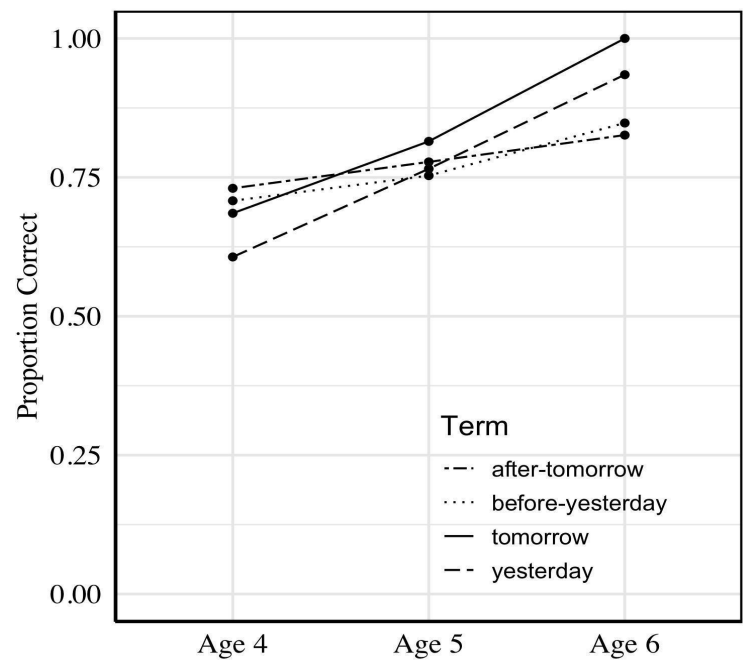

Figure 3: Proportion of children $(N=219$ across language groups), who correctly placed items in the past or the future.

\section{Precise Knowledge}

Next, to assess children's knowledge of the precise meaning of each term, we asked whether language (German vs. English), age, and/or item predicted children's correct placement. All three factors significantly improved the fit of the model: German speakers outperformed English speakers $\left(\beta=0.80, p=.005 ; \chi^{2}(1)=8.03, p=.005\right)$; older children outperformed younger children $\left(\beta=1.44, p<.001 ; \chi^{2}(1)=\right.$ $71.9, \mathrm{p}<.001)$; and performance differed by item $\left(\chi^{2}(3)=\right.$ $78.7, p<.001){ }^{6}$

Follow-up item comparisons within each language group using Wilcoxon signed-rank tests revealed that 4-year-olds from both language groups were significantly more accurate on tomorrow compared to before-yesterday (English: $p=$ .013; German: $p<.001$ ) and after-tomorrow (English: $p=$ .005 ; German: $p=.009$ ). English-speaking 5- and 6-year-olds

\footnotetext{
${ }^{6}$ We did not find evidence of an interaction; the model failed to converge when the interaction term was included. When random
}

were significantly more accurate on yesterday (English: $p \mathrm{~s}<$ .015 ) and tomorrow (English: $p \mathrm{~s}<.015)$ than on beforeyesterday (see Figure 4). No other item-level comparisons were significant, including comparisons of tomorrow and yesterday in all age groups.

In light of the previous analysis suggesting that distal terms might have been more difficult for the youngest children in both languages, we next considered whether language, temporal location (proximal vs. distal), age, or their interaction predicted children's correct placement. All three factors significantly improved the fit of the model, and there was no evidence of an interaction: older children were more likely to place terms in the correct square than younger children $\left(\beta=1.41, p<.001 ; \chi^{2}(1)=72.73, p<.001\right)$; the proximal terms that were lexicalized in both languages (yesterday/tomorrow) were easier for children to identify than the distal terms (after-tomorrow/before-yesterday; $\beta=$ $\left.1.62, p<.001 ; \chi^{2}(1)=71.19, p<.001\right)$; and German speakers were better at both distal terms that are only lexicalized in German, and proximal terms that are lexicalized in both languages $\left(\beta=0.78, p=.004 ; \chi^{2}(1)=8.00, p=.004\right)$.

We also conducted an exploratory analysis considering whether language, the deictic status (past vs. future) of the item, age, or their interaction predicted children's correct placement. All three factors improved the model fit and we found no evidence of an interaction: older children were more likely than younger children to place items in the correct squares $\left(\beta=1.24, p<.001 ; \chi^{2}(1)=74.3, p<.001\right)$; future terms (tomorrow/after-tomorrow) were easier for children to identify than past terms (yesterday/ before-yesterday; $\beta=$ $\left.0.27, p=.001 ; \chi^{2}(1)=10.7, p=.001\right)$; and German speakers were better at both future terms and past terms $(\beta=0.32, p<$ $\left..001 ; \chi^{2}(1)=7.88, p=.004\right)$.

\section{General Discussion}

We conducted a cross-linguistic comparison to examine how different features of temporal language impact time-word learning. To our knowledge, this is the first study to investigate the acquisition of deictic time words in both German- and English-speaking children. Using a stickerplacement task, we showed that German-speaking 3- to 7year-olds were more likely than age-matched Englishspeakers to have precise meanings for both distal terms that are lexicalized only in German (e.g., übermorgen/the day after tomorrow) and proximal terms that are lexicalized in both languages (e.g., gestern/yesterday). Importantly, this was true despite the finding that a greater number of Englishspeaking children were able to recite the days of the week. This result suggests that German-speakers were unlikely to have a general advantage on the task and, instead, that the presence of additional temporal terms in the lexicon might specifically help to constrain children's early time-word meanings.

effects were removed from the model, all three factors still improved model fit and there was no evidence of an interaction. 

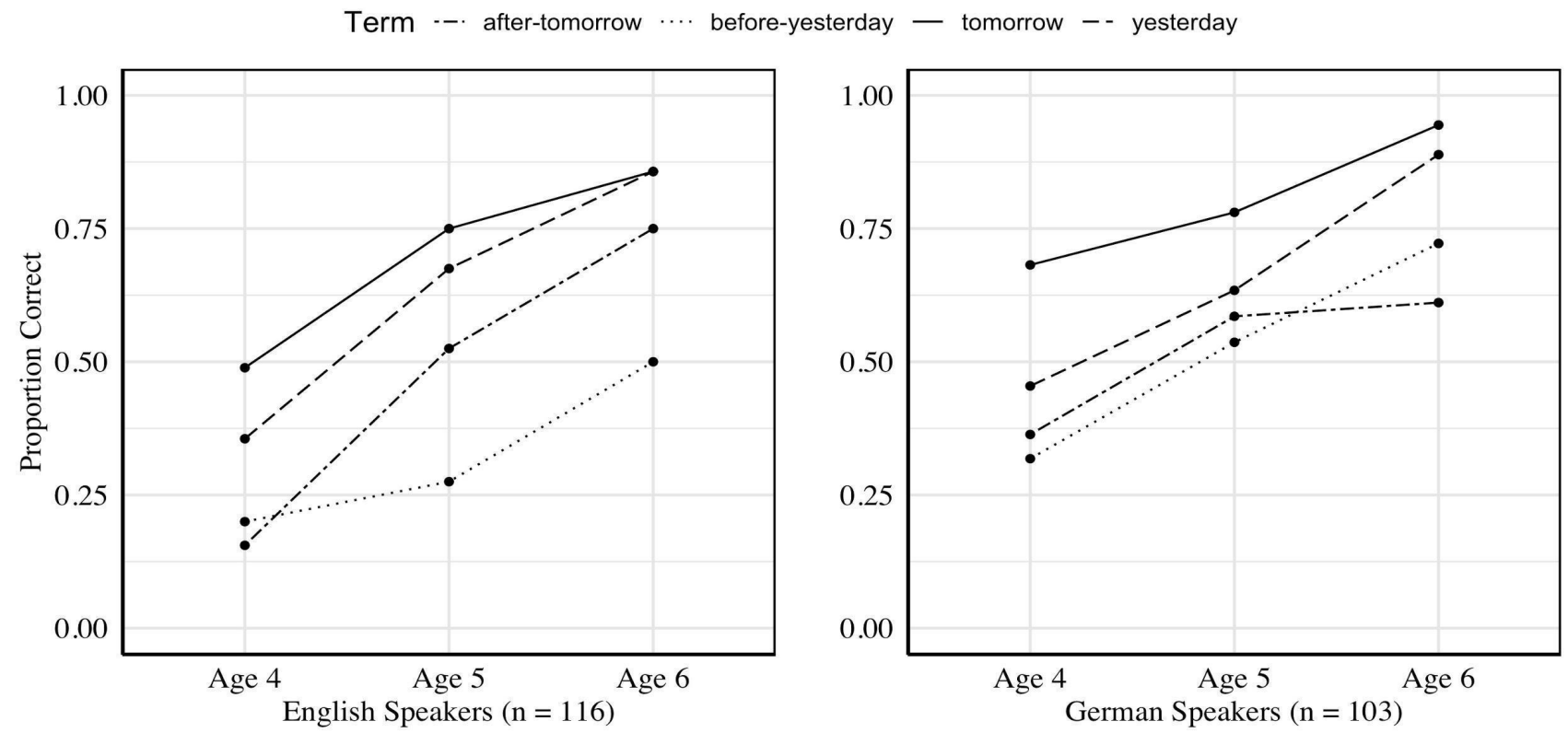

Figure 4: Proportion of English- and German-speaking children, by age, who placed each item in the correct square.

Although German-speaking children were more likely to have precise meanings for time words, we did not find an effect of language on children's acquisition of the deictic status of these terms (e.g., knowing yesterday refers to a time in the past). This is consistent with previous findings in English that different facets of meaning are acquired separately, and that knowledge of deictic status precedes the acquisition of adult-like meanings of time words (Tillman et al., 2017). The lack of a language group difference in children's knowledge of deictic status is also consistent with the idea that children were relying on linguistic cues to learn a facet of meaning that did not differ greatly across languages. In particular, both English and German contain tense markings on verbs that could be sufficient to support inferences about the deictic status of time words.

Why might having additional time words available in the lexicon help German-speaking children acquire precise meanings for time words, including those present in both languages? Prior work suggests that children's initial meanings of time words, including both duration words and deictic time words, are relational in nature. Despite using time words inaccurately for several years, children as young as age 4 know that time words form a common semantic domain (Shatz et al., 2010), and by age 5 they also exhibit an understanding of their relative ordering within that domain (e.g., second < minute < hour; (1) last week, (2) yesterday, (3) today; Tillman \& Barner, 2015; Tillman et al., 2017). Such findings indicate that prior to acquiring their precise meanings, children's knowledge of time words is constrained by their knowledge of other words in the set.

While our results are consistent with the possibility that simply having more time words in the lexicon is better, this seems unlikely to be a general rule. After all, having to learn a new word for every possible temporal location would not make matters easier for children. Another possibility is that differences between English- and German-speaking children's early understanding of yesterday and tomorrow is related to the difference in the proximity of their "nearest neighbors" in the set of temporal terms. In English, the closest temporal alternative to yesterday (that does not require an entire phrase to specify) is last week, which is typically much farther away from yesterday as vorgestern is from gestern. ${ }^{3}$ A closer alternative word may help German speakers restrict the scope of gestern at an age when English speakers are still overextending yesterday to inaccurately refer to more distant times in the past. This pattern is similar to findings in other semantic domains, such as color, in which meanings are initially overextended and narrow as alternative terms are acquired (Wagner et al., 2013).

Given that children in our German- and English-speaking samples were growing up in different cultural contexts, it remains possible that German speakers showed an advantage for some reason unrelated to differences in the lexicons. For example, perhaps German speakers learn about the calendar system before English speakers do. However, arguing against this idea, we found that they were far less likely than English speakers to be able to recite the days of the week. Moreover, formal schooling typically begins earlier in the United States than in Austria, suggesting that English-speakers may have more exposure to the calendar system than German-speakers of the same age. In future work, we plan to conduct additional measures, such as a general language assessment, to examine other possible contributors to the between-group differences. 
The present results suggest that cross-linguistic comparison is a fruitful avenue for exploring how temporal terms and concepts are learned. In addition to varying in the number of time words they contain, languages across the world vary in which aspects of meaning those words encode. For example, Yélî Dnye, an indigenous language spoken offshore from Papua New Guinea, contains terms for up to 10 days in the past or future (Levinson \& Majid, 2013) and thus contains (many) more terms for precise days than does German. In contrast to English and German, some time words in Urdu specify remoteness from the present but do not specify deictic status (e.g., "kal" refers to a time period that is exactly one day away from the present, in either the past or the future; Schmidt \& La Schmidt, 1999). Other languages, like Mandarin Chinese, do not have a morphological tense system and instead rely on adverbials, aspect particles, and situational context to communicate the past/future status of events. These cross-cultural differences in language are important to consider in ongoing investigations of how temporal syntax and other linguistic cues influence children's acquisition of temporal words and concepts. For example, if tense is a useful cue for bootstrapping the acquisition of deictic time words, we might predict earlier acquisition of these words by English- and German-speaking children compared to Mandarin-speaking children. (Tillman et al., 2015; see also Zhang \& Hudson, 2018 for discussion). We plan to explore additional cross-linguistic comparisons in future work.

\section{Acknowledgments}

Thank you to Cole Dougherty and Austin Thought Lab research assistants Shaurya Aggarwal, Alexis Belmares, Tayler Fennell, Bela Gadgil, Amal Hashmey, Christina Howell, Vanessa Jones, Tayler Mancillas, Michael Price, Paige Wilson, and the Vienna Children's Studies research team, especially Josephine Funke and Andrea Morgenbesser, for their assistance with subject recruitment and data collection. We also want to thank the many children and families who participated, without whom this research would not be possible.

\section{References}

Ames, L. B. (1946). The development of the sense of time in the young child. $J$ Genet Psychol, 68, 97-125.

Bates, D., Mächler, M., Bolker, B., \& Walker, S. (2015). Fitting linear mixed-effects models using lme4. $J$ Stat Softw, 67, 1-48.

Dryer, Matthew S. \& Haspelmath, Martin (eds.) 2013. The World Atlas of Language Structures Online. Leipzig: Max Planck Institute for Evolutionary Anthropology.
Fox, J. and Weisberg, S. (2019) An R Companion to Applied Regression, Third Edition, Sage.

Frank, M. C., Braginsky, M., Yurovsky, D., \& Marchman, V. A. (2017). Wordbank: An open repository for developmental vocabulary data. J Child Lang, 44, 677.

Friedman, W. J., \& Kemp, S. (1998). The effects of elapsed time and retrieval on young children's judgments of the temporal distances of past events. Cogn Dev, 13, 335-367.

Grant, J. B., \& Suddendorf, T. (2011). Production of temporal terms by 3-, 4-, and 5-year-old children. Early Child Res $Q, 26,87-95$.

Harner, L. (1975). Yesterday and tomorrow: Development of early understanding of the terms. Dev Psychol, 11, 864.

Hudson, J. A., \& Mayhew, E. M. (2011). Children's temporal judgments for autobiographical past and future events. Cogn Dev, 26, 331-342.

Levinson, S. C., \& Majid, A. (2013). The island of time: Yélî Dnye, the language of Rossel Island. Front. Psychol., 4, 61.

Schmidt, R. L., \& La Schmidt Ruth. (1999). Urdu: An essential grammar. London: Routledge.

Shatz, M., Tare, M., Nguyen, S.P., \& Young, T. (2010). Acquiring non-object terms: The case for time words. J Cogn Dev, 78, 57-77.

Swift, M. D. (2008). Time in child Inuktitut: A developmental study of an Eskimo-Aleut language (Vol. 24). Walter de Gruyter.

Tillman, K., A., \& Barner, D. (2015). Learning the language of time: Children's acquisition of duration words. Cogn Psychol, 78, 57-77.

Tillman, K. A., Cheung, P., Tulagan, N., \& Barner, D. (2015). Do some languages tell time better than others? Acquisition of time words in English- and Chinesespeaking children. Cognitive Development Society biennial meeting.

Tillman, K. A., Marghetis, T., Barner, D., \& Srinivasan, M. (2017). Today is tomorrow's yesterday: Children's acquisition of deictic time words. Cogn Psychol, 92, 87100.

Wagner, K., Dobkins, K., \& Barner, D. (2013). Slow mapping: Color word learning as a gradual inductive process. Cogn, 127(3), 307-317.

Weist, R. M., Atanassova, M., Wysocka, H., \& Pawlak, A. (1999). Spatial and temporal systems in child language and thought: A cross-linguistic study. First Language, 19, 267-308.

Zhang, M. \& Hudson, J.A. (2018). Children's understanding of yesterday and tomorrow. J Exp Child Psychol, 170, 107133. 